\title{
Metabolic pathways and abnormalities
}

Rhys D. Evans ${ }^{1}$

Lisa C. Heather ${ }^{2}$

${ }^{1}$ Reader in Metabolic Biochemistry

University of Oxford

Department of Physiology, Anatomy, and Genetics

Sherrington Building

South Parks Road

Oxford OX1 3PT

U.K.

${ }^{2}$ Diabetes UK RD Lawrence Fellow \& Lecturer in Biochemistry

University of Oxford

Department of Physiology, Anatomy, and Genetics

Sherrington Building

South Parks Road

Oxford OX1 3PT

U.K.

T: +44 (0) 1865272445

F: +44 (0) 1865282272

E: rhys.evans@dpag.ox.ac.uk 


\section{ABSTRACT}

Metabolism describes the series of chemical reactions which are concerned with the provision of energy to biological systems. They may be divided into reactions involved in energy yield (catabolism: demand exceeds supply), and energy storage (anabolism: supply exceeds demand). Regulation of these pathways is critical for homeostasis, and derangements in metabolism are seen in a wide variety of pathological processes. Understanding metabolism is key to the treatment of many diseases, notably diabetes, as well as underpinning clinical nutritional support.

Key words: Metabolism, carbohydrates, lipids, proteins, diabetes 
Metabolism is derived from the Greek "to change", and describes the series of biochemical reactions that provide the body with the energy it requires to maintain biological functions (e.g. biosynthesis, maintenance of ionic gradients, muscle contraction, heat generation). This energy must ultimately be derived from food. The rate of energy production is measured under basal conditions - "basal metabolic rate" (BMR), and is affected by many factors, including muscle contraction, food ingestion, size, gender, age, temperature, sepsis, and several hormones, including thyroid hormones and catecholamines. The metabolic rate can be estimated by measuring oxygen consumption $\mathrm{VO}_{2}$; indirect calorimetry).

The process of converting excess energy-rich substrate precursors into complex energy storage molecules is termed anabolism, whilst processes degrading substrates to mobilise biologically usable energy, are termed catabolism. Imbalance of these pathways leads to cachexia or obesity. Tissues have specialised metabolic functions - e.g. adipose tissue stores energy, muscle oxidises substrate, lactating mammary gland exports substrate. The liver is a metabolic "transformer" that regulates substrate supply between tissues, and pancreas detects and signals nutritional status.

Metabolic energy is carried in two main forms: 1. "high energy" phosphate groups including ATP, GTP and creatine phosphate, and 2. Hydride ion (electron) carriers such as NADH, $\mathrm{FADH}_{2}$ and NADPH. These energy molecules are used in chemical reactions throughout the cell that would not occur without external energy input, because they are energetically unfavourable. Besides carrying energy in metabolic pathways, the energy charge and redox potential are major regulators of metabolism.

\section{Energy substrates}

Energy is derived from three groups of energy-rich compounds: carbohydrates, lipid (fats) and proteins (amino acids) (Figure 1). Carbohydrates are soluble, fast and easy to transport, relatively non-toxic, and some energy can be derived from them anaerobically during hypoxia or ischaemia when oxygen is limited. However, their water solubility means that in storage form as glycogen they retain significant amounts of water and, therefore, only limited amounts are stored. In addition, carbohydrates are partially oxidised and hence do not contain as much energy as lipids. By contrast, lipids are highly reduced and very energy-dense, hence they function as the principal energy store for free-living animals and are major energy providers to most tissues. However, their water-insolubility makes 
lipids slow to mobilise, and unlike carbohydrates they cannot yield energy anaerobically, so cannot be used by erythrocytes and renal medulla. In addition, they cannot cross the blood-brain barrier so cannot be used by the CNS. Because they are more reduced, relatively more oxygen is required to extract energy from lipids (2.8 ATP/O 2 ) compared to carbohydrates (3.7 ATP/O $/ \mathrm{O}_{2}$ ) and this may be critical in high work-load oxygen-challenged tissues such as myocardium and exercising skeletal muscle. Also, lipids in the form of nonesterified fatty acids are amphipathic (detergent-like) and hence toxic. Amino acids have similar energy yields to carbohydrates, similar solubility and most can be converted to glucose. Under conditions of carbohydrate depletion (e.g. starvation) certain proteins can be broken down to yield amino acids for conversion into carbohydrates to supply glucosedependent tissues. Although proteins are not stored specifically to supply energy, they can act as a virtual carbohydrate supply in catabolic states of carbohydrate exhaustion.

The three major substrate groups converge at a common intermediate, acetyl-CoA. Mitochondrial acetyl-CoA can enter the tricarboxylic acid (TCA) cycle and be completely oxidised to $3 \mathrm{NADH}, 1 \mathrm{FADH}_{2}, 1 \mathrm{GTP}$ and $2 \mathrm{CO}_{2}$. The hydrogen carriers enter the electron transport chain in addition to oxygen, and result in the generation of large amounts of ATP via oxidative phosphorylation.

Pancreas is the key organ detecting metabolic status. Pancreatic $\beta$-cells sense high blood glucose and release insulin in response (this mechanism is affected by sulphonylurea drugs). Pancreatic $\alpha$-cells release glucagon in response to low blood glucose concentration - their glucose detection mechanism may be analogous to $\beta$-cells and/or they may respond to $\beta$-cell status by an autocrine/paracrine mechanism. Carbohydrate and lipid utilisation are reciprocally related (Randle cycle), a mechanism partly orchestrated by insulin.

\section{CARBOHYDRATE METABOLISM}

Carbohydrate metabolism centres around the hexose sugar glucose $\left(\mathrm{C}_{6} \mathrm{H}_{12} \mathrm{O}_{6}\right)$. Following uptake into the cell by glucose transporters (GLUT), glucose is rapidly phosphorylated to glucose-6-phosphate (G6P) by the enzyme hexokinase (liver and pancreas: glucokinase); G6P is a central hub in carbohydrate metabolism and can used for glycolysis and glycogen synthesis (glycogenesis), and it may also be derived from glycogen breakdown (glycogenolysis) and from non-carbohydrate precursors (gluconeogenesis), depending on tissue and prevailing metabolic state. 


\section{Glycogen}

Carbohydrate is stored in limited amounts as cytoplasmic glycogen granules in most tissues, as an energy resource available within the tissue (and hence independent of blood supply) for rapid utilisation when required. Glycogen is a polymer of glucose that glycogen synthase assembles into a linear chain, but every 8-10 residues a branch point is introduced. This produces a highly branched tree-like structure with many free ends, which enable rapid glucose release during glycogenolysis by the enzyme glycogen phosphorylase. In tissues which store glycogen for their own utilisation (e.g. muscle), the G6P generated by glycogenolysis undergoes glycolysis for energy production, however, in liver the G6P is dephosphorylated by glucose-6-phosphatase into free glucose, which is released into the blood stream to maintain blood glucose levels. Liver stores about $100 \mathrm{~g}$ of glycogen, enough to supply the body for about 12 hours, whereas skeletal muscle stores about $350 \mathrm{~g}$ of glycogen, sufficient for $\sim 70$ minutes of muscle contraction.

\section{Glycolysis}

Glucose is cleaved into pyruvate by glycolysis in the cytosol of all cells (Figure 2), and generates energy without the use of oxygen. One molecule of glucose yields 2 molecules of pyruvates, 2 NADH and 2 ATP, the latter via substrate level phosphorylation. Pyruvate can be imported into the mitochondria and decarboxylated to acetyl CoA, remain in the cytosol and be reduced to lactate, or be transaminated to amino acids. The fate is determined by the tissue, oxygen availability and circulating hormones. In muscle, glycolysis splits glucose in order to provide energy, but in liver, excess glucose is broken down by glycolysis to pyruvate, then acetyl-CoA, and used for lipid synthesis (Figure 1). Glycolysis is tightly regulated by hormonal and metabolic signals: in liver, insulin stimulates, and glucagon inhibits, glycolysis. Glycolysis is linked to the energy status of the cell via allosteric effects of AMP, ATP and citrate.

\section{Gluconeogenesis}

Gluconeogenesis is typically active in catabolic states, e.g. post-prandial/starvation, exercise, and mainly occurs in the liver (in keeping with its role in maintaining blood glucose levels) with some limited activity in kidney. It allows the body to make new glucose when dietary carbohydrate sources are limited, and is regulated by hormones such as glucagon, and by the supply of substrates. The pathway is not simply a reversal of glycolysis, as several reactions of glycolysis are irreversible, but many of the steps are 
shared by both pathways. The substrate for gluconeogenesis is pyruvate, derived from transamination of the amino acid alanine or from re-oxidation of lactate produced from anaerobic metabolism. Glycerol, derived from triglycerides (TAG), can also be used for glucose synthesis, and hence breakdown of storage lipids does yield a small amount of carbohydrate.

\section{Lactate and ethanol metabolism}

In tissues with mitochondria and in the presence of oxygen, $\mathrm{NADH}$ from glycolysis is reoxidised to $\mathrm{NAD}^{+}$by the electron transport chain with generation of $A T P$. In the absence of mitochondria (e.g. erythrocytes) or in ischaemic/hypoxic states, NAD+ must be regenerated from $\mathrm{NADH}$ in the cytosol, by linking pyruvate reduction to lactate by lactate dehydrogenase (LDH) to allow glycolysis to continue (Figure 2). Lactate accumulates and glycolysis proceeds, providing limited ATP production by anaerobic metabolism: "homolactic fermentation". Tissues lacking oxygen (e.g. exercising muscle) or mitochondria (erythrocytes) export lactate to the liver where it is re-oxidised to pyruvate, which then undergoes gluconeogenesis, reforming glucose for re-export to muscle or erythrocytes: the Cori cycle. This cycle likely operates in tumour cells which are highly glycolytic. In the clinical context, elevated blood lactate is a common finding in critically ill patients. Hyperlactataemia/lactic acidosis (blood [lactate] $>2.5 \mathrm{mM}$ ) may be due to increased peripheral (extrahepatic) production (e.g. tissue ischaemia/hypoxia) but may also be due to decreased central (hepatic) gluconeogenesis or blood, and is an early sign of mesenteric/hepatic ischaemia.

Certain organisms (e.g. yeasts) have an alternative strategy to regenerate $\mathrm{NAD}^{+}$for glycolysis - alcoholic fermentation. Here, pyruvate is decarboxylated to acetaldehyde, which is then reduced to ethanol by alcohol dehydrogenase, linked to the oxidation of $\mathrm{NADH}$, regenerating $\mathrm{NAD}^{+}$(Figure 2). Ethanol accumulates, and inhibits competing microorganisms. When ethanol is ingested by humans, its metabolism has multiple effects on the $\mathrm{NAD}^{+}: \mathrm{NADH}$ ratio (redox potential). In the liver, ethanol is oxidised to acetaldehyde by alcohol dehydrogenase, and acetaldehyde is further oxidised to acetate by aldehyde dehydrogenase, both enzymes generating NADH (and potentially reactive oxygen species). The acetate is converted into acetyl-CoA, providing an abundant energy source. However, the high levels of NADH consequently inhibit oxidation of lactate to pyruvate, limiting the availability of the gluconeogenic precursor and causing a mild metabolic lactic acidosis. The result is decreased gluconeogenesis and hypoglycaemia. Furthermore, the 
TCA cycle and fatty acid $\beta$-oxidation are inhibited, whilst lipogenesis is increased, (increased acetyl-CoA), leading to hepatic lipid accumulation and alcoholic fatty liver.

\section{Pentose phosphate pathway}

G6P is also used in the cytosolic pentose phosphate pathway (PPP). The pathway generates NADPH, and the pentose (5-carbon) sugar ribulose-5-phosphate, which is used for synthesis of nucleotides and aromatic amino acids. NADPH provides energy for certain anabolic reactions, such as lipogenesis, and also maintains the antioxidant glutathione in its active reduced (active) form (GSH). The initiating step of PPP reduces $\mathrm{NADP}^{+}$to NADPH and is catalysed by glucose-6-phosphate dehydrogenase (G6PDH). Deficiency of this enzyme is common in equatorial regions. It is an X-linked condition and many variants of G6PDH deficiency occur. It is associated with oxidative damage to the erythrocyte causing haemolytic anaemia, which may be induced by various drugs and chemicals, including methylene blue, and ingested fava beans (favism). Lack of NADPH secondary to low G6PDH activity results in low levels of reduced glutathione and increased oxidative damage to erythrocytes, denatured haemoglobin appearing as blister cells and Heinz bodies. G6PDH mutations have probably been tolerated in evolution because lack of NADPH-derived anti-oxidant activity in erythrocytes inhibits the malaria parasite.

\section{Regulation of carbohydrate metabolism}

Insulin is the major anabolic signal for carbohydrate metabolism. Pancreatic $\beta$-cells secrete insulin in response to increased blood glucose, which stimulates anabolic pathways such as glycogenesis and glycolysis, which lowers blood glucose. Insulin also inhibits catabolic pathways such as glycogenolysis and gluconeogenesis. Sympathetic activation and several hormones, including catecholamines, cortisol and growth hormone, stimulate hepatic glycogenolysis and gluconeogenesis, but glucagon is the major catabolic signal, raising blood glucose by stimulating hepatic glucose production and inhibiting the reciprocal anabolic pathways. Hepatic gluconeogenesis is inhibited by the biguanide metformin, decreasing hepatic glucose production in diabetes.

\section{LIPID METABOLISM}

Fatty acids are used for energy production in oxidative tissues. Since they are amphipathic and toxic, their plasma concentration does not rise to more than $\sim 0.5 \mathrm{mM}$ physiologically and they are transported bound to albumin ("free" fatty acids are more correctly termed 
non-esterified fatty acids (NEFA)). Esterification of three fatty acids to glycerol yields the very hydrophobic TAG. TAG transport in the aqueous plasma requires it to be carried in the hydrophobic core of TAG-rich lipoproteins (TGRLP).

\section{Lipid Mobilisation}

Lipid mobilisation occurs during catabolic states such as fasting, starvation and exercise. Adipose tissue stores the greatest amount of TAG $(\sim 15 \mathrm{~kg})$, which undergoes lipolysis by the action of lipase enzymes (including hormone sensitive lipase, HSL) releasing three fatty acids (FA) and one glycerol into the circulation for use elsewhere in the body. Glycerol is utilised by the liver for gluconeogenesis; the fatty acids are utilised by oxidative tissues for energy. Other tissues store TAG in lipid droplets as an intracellular energy resource for their own utilisation.

NEFA uptake from the plasma involves both diffusion across the cell membrane as well as facilitated uptake via CD36/FAT (FA translocase) and FA binding and transport proteins Following uptake, FAs may be re-esterified in the cytosol to intracellular triglycerides or migrate to the mitochondria for oxidation. However, long chain FA-CoA cannot cross the highly selective inner mitochondrial membrane, therefore, the FA is transported across on the carnitine shuttle, initiated by carnitine acyl transferase-1 (CAT-1). CAT-1 is inhibited by malonyl-CoA, the first committed intermediate of lipogenesis, a mechanism preventing simultaneous FA synthesis and breakdown, and a major regulatory mechanism of FA oxidation.

\section{$\beta$-oxidation}

FA-CoA within the mitochondria now undergoes $\beta$-oxidation. The $\beta$-carbon of the FA chain is attacked and a 2-carbon segment of the FA chain is released as acetyl-CoA. This oxidative cycle is repeated until the entire FA chain has been broken down to multiple acetyl-CoA, NADH and FADH 2 . The acetyl-CoA undergoes further oxidation in the TCA cycle; all the $\mathrm{NADH}$ and $\mathrm{FADH}_{2}$ generated are then oxidised by the electron transport chain, yielding large amounts of ATP. Medium chain acyl-coenzyme A dehydrogenase (MCAD) deficiency impairs the $\beta$-oxidation of fatty acids within the mitochondria, limiting their use as a fuel in oxidative tissues and increasing the dependence on glucose metabolism for ATP production.

\section{Ketone bodies}


In the liver, acetyl-CoA derived from $\beta$-oxidation can also be used for ketone body synthesis (ketogenesis). The ketone bodies, acetoacetate and $\beta$-hydroxybutyrate, are water soluble transportable forms of acetyl-CoA, which can be used by the brain and other oxidative tissues as glucose-sparing fuels. Ketogenesis occurs uniquely in the liver, however, liver lacks the pathway for ketone body utilisation (ketolysis), preventing futile substrate cycling. An intermediate of ketogenesis is hydroxymethylglutaryl CoA (HMGCoA), which can be converted to cholesterol by HMG-CoA reductase, the enzyme inhibited by the statin class of drugs. Acetoacetate undergoes spontaneous decarboxylation to acetone, which probably has no physiological function in humans but is volatile and excreted in the breath, with a characteristic sweet-smelling odour present in diabetic ketoacidosis.

\section{Lipid synthesis and lipoprotein metabolism}

Excess acetyl-CoA derived from surplus carbohydrates and amino acids is assembled into fatty acids for energy storage (lipogenesis) in the cytosol of liver and adipose tissue (Figure 1). The initiating step involves generation of malonyl-CoA from acetyl-CoA by acetyl-CoA carboxylase (ACC), and is highly regulated. The malonyl group is the donor for fatty acid synthetase (FAS), a multicatalytic polypeptide which elongates the growing fatty acid chain by 2 carbons in a repeated cycle using NADPH for energy. Whilst $\beta$-oxidation occurs in mitochondria, lipogenesis occurs in the cytosol, an example of intracellular compartmentation limiting futile substrate cycling of two opposing pathways. Three FAs are then esterified to glycerol phosphate to form TAG. TAG synthesised in liver must next be exported to adipose tissue for storage.

TAG must be transported in the plasma within specialised carrier particles - lipoproteins. The TAG-rich lipoproteins (TGRLP) comprise a phospholipid monolayer shell, embedded proteins (apolipoproteins), a hydrophobic core of TAG, esterified cholesterol, and fatsoluble vitamins. Two TGRLPs carry TAG: chylomicrons (CM), synthesised by the intestine from exogenous dietary fat, and very low density lipoproteins (VLDL), synthesised by the liver from endogenous lipids. CM and VLDL deliver TAG to FA-utilising tissues that express the enzyme lipoprotein lipase (LPL), which is tethered to the luminal surface of the endothelium. In type I hyperlipoproteinaemia (chylomicronaemia) syndrome, an autosomal recessive mutation of LPL, plasma TGRLP-TAG cannot be cleared and very high ( $>50 \mathrm{mM}$ ) plasma TAG levels result. More rarely, a mutation in apo-CIl protein expressed on FAutilising tissues can cause a similar clinical picture. 
Following LPL action, the lipoprotein shrinks resulting in a smaller, denser, TAG-depleted particle named a "remnant particle". The chylomicron remnant is recycled in the liver; the VLDL remnant particle is termed low-density lipoprotein ( $L D L)$, and continues in the circulation to deliver its remaining core lipid - cholesterol ester - to peripheral tissue through a lipoprotein receptor-mediated uptake mechanism, resulting in the entire LDL particle being endocytosed into the target cell for cholesterol release. This mechanism is termed "forward cholesterol transport". Excess cholesterol is transported back from peripheral tissues to the liver for excretion by the "reverse cholesterol transport" pathway: nascent high-density lipoprotein (HDL) particles in the plasma assimilate cholesterol from extrahepatic tissues, the cholesterol-enriched HDL particle being removed by the liver and the cholesterol excreted in the bile. Defects in lipoprotein receptor expression, for example lack of functional LDL receptor in familial hypercholesterolaemia (Fredrickson type II hyperlipidaemia), are characterised by an inability to remove LDL cholesterol from the circulation, resulting in very high plasma LDL-cholesterol levels $(>10 \mathrm{mM})$ and accelerated atherosclerosis.

\section{Regulation of lipid metabolism}

The anabolic state is signalled by insulin: hepatic lipogenesis, TAG and cholesterol synthesis are stimulated, whilst ketogenesis is inhibited. In adipose tissue, insulin stimulates LPL, enhancing plasma TAG uptake from TGRLPs, and suppresses TAG lipolysis, thereby lowering plasma NEFA concentrations. Conversely, several catabolic "counter-regulatory" hormones and signals stimulate lipid mobilisation and breakdown catecholamines, increased sympathetic activity, and adrenocorticotrophic hormone (ACTH) stimulate adipose lipolysis, increasing plasma NEFA levels.

\section{AMINO ACID METABOLISM}

Typical dietary protein intake is $\sim 100 \mathrm{~g} / \mathrm{day}$, whilst the $\sim 10 \mathrm{~kg}$ of body protein is turned over at $\sim 300 \mathrm{~g} /$ day $(\sim 3 \%)$. Dietary proteins are absorbed as amino acids and small peptides into the portal circulation, with enterocytes and hepatocytes oxidising some amino acids as their main substrate. Amino acids are also derived from proteolysis of endogenous proteins, and some ("non-essential") can be synthesised from intermediary metabolites or from other amino acids. In contrast, "essential" amino acids cannot be synthesised by humans and must be obtained from the diet. Unlike carbohydrates and lipids, amino acids contain nitrogen in the form of an amino group and this must be removed (deamination) 
before the remaining carbon skeleton (2-oxoacid) can undergo further metabolism (Figure 3). Deamination of amino acids produces ammonia $\left(\mathrm{NH}_{3}\right)$, which is highly toxic and must be either excreted directly into the urine by the kidney, or converted into relatively nontoxic urea by the urea (ornithine) cycle in the liver.

\section{Nitrogen disposal}

Deamination of amino acids is achieved by two types of reaction acting together. In transamination, the $\alpha$-amino group from one amino acid is transferred to another 2-oxoacid (carbon skeleton), forming its corresponding amino acid (i.e. aa-1 + 2-oxoacid-2 $\leftrightarrow 2$ oxoacid-1 + aa-2). For most amino acids undergoing transamination the amino acceptor is a-ketoglutarate ( a TCA cycle intermediate), producing the carbon skeleton of the donor amino acid and glutamate. Hence $\alpha$-ketoglutarate "funnels" the various amino acids into glutamate (Figure 4). Alanine aminotransferase (transaminase; ALT) transfers the amino group of alanine to $\alpha$-ketoglutarate, forming pyruvate and glutamate. Alanine is a key transport amino acid in the blood, hence this enzyme is important for inter-tissue amino acid flux. Aspartate aminotransferase (transaminase; AST) transfers the amino group of aspartate to $\alpha$-ketoglutarate, forming oxaloacetate and glutamate but this enzyme usually works in the reverse direction, converting glutamate (from funnelled amino acids, above) into aspartate, which is required to donate a second $\mathrm{N}$-atom to the urea cycle. Since ALT and AST are both intracellular enzymes and widespread, necrosis of many tissues releases them to plasma, but they are commonly used to diagnose hepatocellular damage. The second type of deamination reaction is oxidative deamination: following transamination, the glutamate undergoes direct, oxidative, deamination, by glutamate dehydrogenase, regenerating $\alpha$-ketoglutarate and producing $\mathrm{NH}_{3}$. The ammonia is then detoxified to urea in the urea cycle, and carbon skeletons undergo intermediary metabolism (Figure 4).

The urea cycle occurs in the liver. Urea $\left(\mathrm{CO} .\left(\mathrm{NH}_{2}\right)_{2}\right)$ contains two nitrogen atoms: one derives from $\mathrm{NH}_{3}$ via oxidative deamination of glutamate, the other from aspartate via transamination by AST. Each of the six enzymes of the urea cycle may be functionally mutated, impairing ammonia disposal. Ornithine transcarbamylase (OTC) is a mitochondrial urea cycle enzyme which synthesises citrulline from ornithine and carbamoyl phosphate (the latter formed from ammonia and bicarbonate). OTC deficiency is the commonest disorder of the urea cycle and is characterised by high ammonia concentrations, causing ataxia, lethargy and death, reflecting the extreme neurotoxicity of 
$\mathrm{NH}_{3}$. The mechanism of the severe neurotoxicity of ammonia is not fully understood, but excess free ammonia in the CNS may cause reversal of glutamate dehydrogenase, with glutamate formation - this depletes a-ketoglutarate, a key TCA cycle intermediate and hence depletes ATP. Since glutamate is an excitatory neurotransmitter, this may also account for the observed effects on neural function.

\section{Metabolism of carbon skeleton}

Following deamination, the remaining carbon skeleton enters the common metabolic pool. All amino acids ultimately yield just seven products of intermediary metabolism: pyruvate, a-ketoglutarate, succinyl-CoA, fumarate, oxaloacetate, acetyl-CoA and acetoacetyl-CoA. The first five of these represent $\geq 3$ carbons, hence amino acids producing these metabolites can be used for glucose synthesis ("glucogenic"): it is this property that confers on proteins the ability to act as a carbohydrate reserve. The acetyl-CoA and acetoacetyl-CoA, however, yield two (or two-equivalent) carbons, and amino acids which produce them cannot be used for gluconeogenesis - they can be directly oxidised in the TCA cycle, undergo lipogenesis or be used to synthesise ketone bodies ("ketogenic").

In alkaptonuria, the gene for the enzyme homogentisate 1,2-dioxygenase is functionally mutated. This enzyme is required for the metabolism of the carbon skeletons of phenylalanine and tyrosine to acetoacetyl-CoA. Lack of enzyme activity results in accumulation of the intermediate homogentisic acid; its metabolite alkapton gives the urine a black appearance when exposed to air. The inborn error of metabolism phenylketonuria is also due to a mutation in this same pathway, but the striking difference in disease severity lies in the different metabolites that accumulate when the pathway is blocked further upstream.

\section{Inter-tissue amino acids flux}

Liver is the site of both ureagenesis (amino-N metabolism) and gluconeogenesis (carbon skeleton metabolism), and removes most dietary amino acids from the portal circulation, together with amino acids derived from proteolysis in extrahepatic tissues. The muscle exports a large amount of its amino acids from proteolysis as alanine, derived from the transamination of multiple amino acids donating their amino group to glycolytically-derived pyruvate. The alanine is transported to the liver, where it is transaminated to reform pyruvate, which undergoes gluconeogenesis to glucose (Figure 4), and the glucose is reexported back to muscle (the glucose-alanine cycle). Amino acids are also exported as 
glutamine, which contains two amino groups and is a major transporter of amino groups. The kidney utilises glutamine, removing the side chain amino group with glutaminase, reforming glutamate and free ammonia; the latter is excreted in the urine as a urinary buffer (conferring buffering capacity without losing substrate).

The three branched-chain amino acids (BCAA) - leucine, isoleucine and valine - make up approximately one third of all amino acids in the body. Dietary BCAAs are not removed from the portal circulation by the liver, appearing in high concentration in the splanchnic blood, where they may also have a role as nutrient signals. They are metabolised in extrahepatic tissue, especially muscle, where they are major sources of nitrogen to maintain pools of glutamine, glutamate, and alanine. All three are transaminated by a single branched-chain aminotransferase, and the resulting branched-chain 2-oxoacids undergo oxidative decarboxylation by a branched chain a-ketoacid dehydrogenase. Absence of this enzyme is responsible for maple syrup urine disease, whereby BCAAs are transaminated to their corresponding branched chain $\alpha$-ketoacids, but absence of the dehydrogenase means these intermediates accumulate, appearing in the urine and giving it its characteristic maple syrup odour.

\section{Regulation of amino acid metabolism}

Insulin is the main anabolic signal for protein metabolism, stimulating protein synthesis and inhibiting proteolysis. Net protein anabolism is also stimulated by muscle training, growth factors, growth hormone and anabolic steroids. Protein catabolism is stimulated by cortisol and thyroid hormones. Amino acids act as nutrient signals in pancreatic $\beta$-cells, modulating insulin secretion.

\section{DIABETES}

Blood glucose and certain amino acids - arginine, leucine - are sensed by pancreatic $\beta$ cells; high levels indicate nutrient repletion, and this anabolic state is signalled to the body by insulin release - hence, the pancreas detects glucose whilst the rest of the body then detects insulin. Insulin is the sole anabolic hormone; its actions are opposed by multiple catabolic signals. Consequently, insulin deficiency has severe and pleiotropic metabolic consequences. Inhibition of catabolic pathways by insulin is as critical as stimulating anabolic pathways. In low insulin states, failure to inhibit catabolism, together with lack of anabolic stimulation, causes default to catabolism. In type 1 diabetes mellitus, pancreatic $\beta$-cells are destroyed, so there is no longer any effective glucose sensing mechanism. 
Peripheral tissues interpret lack of insulin as indicating whole body nutrient depletion and respond by becoming catabolic, decreasing substrate uptake and mobilising energy reserves. The liver interprets low insulin as indicating hypoglycaemia, and responds by increasing glucose production via glycogenolysis and gluconeogenesis. The gluconeogenic substrate is alanine, derived from increased proteolysis and muscle wasting. Hence carbohydrate (glycogen) and amino acid (protein) reserves are consumed in an effort to increase substrate provision. Because insulin remains low the catabolic state continues and substrate mobilisation prevails. In addition to increased hepatic glucose production, peripheral glucose utilisation via glucose uptake, glycolysis and glycogenesis is decreased, in an attempt to spare glucose. However, despite the lack of insulin "signalling starvation", the patient is not starving (indeed is still eating) so dietary glucose also enters the circulation, resulting in extreme hyperglycaemia. The excess plasma glucose appears in urine (glycosuria) as the renal tubular reabsorption maximum is exceeded, this carries water osmotically resulting in polyuria, dehydration, thirst, and polydipsia. However, lipid metabolism is also involved as adipose tissue responds to low insulin by increasing TAG lipolysis, with excessive NEFA release into the circulation. The NEFA is utilised by muscle, further decreasing glucose utilisation, and by liver for ketogenesis. The ketone bodies also inhibit glucose utilisation, spill over into the urine (ketonuria), and being acidic cause a metabolic (keto-)acidosis. This is compensated by increased ventilation - Kussmaul breathing - and the volatile 3-carbon acetone produced from acetoacetate may be noticed by its characteristic smell on the breath. 


\section{REFERENCES AND FURTHER READING}

Randle PJ, Garland PB, Hales CN, Newsholme EA. The glucose fatty-acid cycle. Its role in insulin sensitivity and the metabolic disturbances of diabetes mellitus. Lancet. 1963;1:785-789

Hue L, Taegtmeyer $\mathrm{H}$. The randle cycle revisited: A new head for an old hat. Am J Physiol Endocrinol Metab. 2009;297:E578-591

McKnight SL. On getting there from here. Science. 2010;330:1338-1339

Stanley WC, Recchia FA, Lopaschuk GD. Myocardial substrate metabolism in the normal and failing heart. Physiol Rev. 2005;85:1093-1129

Frayn KN. Metabolic regulation. A human perspective. 2010; $3^{\text {rd }}$ edition. Wiley-Blackwell, Chichester 


\section{LEGENDS}

\section{Figure 1}

Energy provision by the 3 key substrate classes. Large storage macromolecules are broken down to intermediate substrates before conversion to acetyl-CoA, the common fuel for TCA (tricarboxylic acid) cycle in the mitochondrion. Acetyl-CoA is oxidised to carbon dioxide and energy yield used in the electron transport chain to produce ATP. Under condition of energy excess, large macromolecules are synthesised for energy storage.

\section{Figure 2}

Carbohydrate metabolism. Ethanol producing pathways (e.g. yeasts) are shown in colour. Both lactate and ethanol production are true fermentation reactions. 1: lactate dehydrogenase 2: alcohol dehydrogenase 3: acetaldehyde dehydrogenase.

\section{Figure 3}

Amino acid metabolism. The metabolic fate of the carbon skeleton depends on where it enters the pathway of intermediary metabolism. TCA: tricarboxylic acid.

\section{Figure 4}

Alanine aminotransferase (ALT) and aspartate aminotransferase (AST) "funnel" amino acids (e.g. from muscle protein) by transamination into glutamate for deamination and urea formation. 